\title{
Guía metodológica para la atención a escolares con dislalia funcional en el subnivel básica elemental
}

\author{
Metodologica guide to the care of schoolchildren with functional dyslalia \\ on the elementary basic sub-leve
}

Autora: Carmen Mindiola Vera. ${ }^{1}$

Recibido: 14-08-2020 / Revisado: 18-09-2020 /Aceptado: 10-10-2020/ Publicado: 06-11-2020

\begin{abstract}
.
DOI: https://doi.org/10.33262/cienciadigital.v4i4.1.1452

The work addresses the need for teachers to prepare to attend schoolchildren with functional dyslalia at the elementary basic sublevel. Its objective was to design a methodological guide that favors literacy learning in boys and girls with functional dyslalia. To support the proposal, surveys, interviews with managers, teachers, schoolchildren, parents, as well as direct observation were carried out. Through the results obtained, it was concluded that there is a lack of preparation for teachers and parents to direct the process of preparing students with functional dyslalia for learning to read and write. A methodological guide is provided with activities that promote learning to read and write. Its application demonstrates the validity of the proposal, aimed at differentiated care, for quality education.
\end{abstract}

Keywords: Functional dyslalia, learning to read and write.

\section{Resumen}

El trabajo aborda la necesidad de preparación de los docentes para la atención a escolares con dislalia funcional en el subnivel básica elemental. Tuvo como objetivo, diseñar una guía metodológica que favorezca el aprendizaje de la lectoescritura en los niños y niñas con dislalia funcional. Para sustentar la propuesta se realizaron encuestas, entrevistas a directivos, docentes, escolares, padres de familia, así como, observación directa, a través de los resultados arrojados se concluyó que existe carencia en la preparación de los docentes y padres de familia, para dirigir el proceso de preparación de los escolares con dislalia funcional, para el aprendizaje de la lectoescritura. Se aporta una guía metodológica con actividades que favorecen el aprendizaje de la lectoescritura. Su aplicación demuestra la validez de la propuesta, orientada a una atención diferenciada, para una educación de calidad.

Palabras clave: Dislalia funcional, aprendizaje de la lectoescritura

\footnotetext{
${ }^{1}$ Centro de Estudios Espíritu Santo, cmindiola@espiritusanto.edu.ec
} 


\section{Introducción.}

\section{Generalidades sobre el lenguaje, sus trastornos e incidencia en Ecuador.}

El lenguaje es la capacidad superior exclusiva del hombre de abstraer y generalizar los fenómenos de la realidad, reflejándola por medio de signos convencionales. El lenguaje es humano por excelencia, siendo esencialmente un hecho psicológico, pero a la vez, un hecho social. Cabanas, (1979)

El habla es uno de los procesos del que se sirve el lenguaje, equivalente a la palabra, a la articulación, a la acción de los órganos fonoarticulatorios. Como resultado se produce la emisión de los fonemas, las palabras, frases y oraciones. Las alteraciones del habla incluyen a los trastornos de la pronunciación, de la fluencia verbal y de la articulación. Las alteraciones en la articulación de los sonidos del habla, se observa de forma evidente en las dislalias. Pérez, (2016)

En Ecuador se trabaja para que el docente esté capacitado para la atención a escolares con necesidades educativas especiales asociadas o no a discapacidad, se busca optimizar la eficacia de la educación donde se identifiquen los problemas desde edades tempranas.

Estudios realizados por Muse, \& Delicia, (2015), demuestran que los trastornos del habla se presentan con mayor frecuencia en infantes entre 6 y 8 años de edad. Uno de estos trastornos se denomina dislalia funcional. Este trastorno tiene una alta incidencia en el proceso de aprendizaje de la lectoescritura, repercutiendo de manera negativa en los niños, ya que influyen y retardan su desarrollo. Lo preocupante es que se ha podido observar que cada vez es más frecuente en las aulas de clases, es por ello que se requiere abordar con mayor profundidad y desde todos los aspectos para poder potenciar y optimizar los procesos de aprendizajes de la lectura y la escritura desde edades tempranas.

Ante esta situación se requiere encontrar vías de solución conducentes a influir en la preparación general de los docentes y familiares para que ayuden a compensar la presencia de dicho trastorno.

\section{La dislalia como trastorno en la articulación.}

La dislalia es un trastorno en la articulación de los fonemas o grupos de fonemas, causado por alteraciones funcionales de los órganos periféricos del habla (lengua, labios, paladar, mejillas y dientes). Es muy frecuentes en la infancia, sobre todo en los primeros años escolares, afectando la pronunciación de cualquier consonante o vocal. Sin embargo, se comparte con (Rodríguez, 2010) al plantear que con una adecuada y oportuna intervención tiende a desaparecer. 
Para lograr el éxito en el proceso de intervención es necesario profundizar en cuáles son las consecuencias de la dislalia funcional, de qué manera repercute en el aprendizaje de la lectoescritura, así como, evaluar que metodología puede contribuir al aprendizaje de la lectoescritura en escolares con dislalia funcional. Concordamos con Loza, (2018), que en su atención debe primar el enfoque personalizado sobre el grupal. Precisamente ahí es donde radica una de las posibles soluciones, porque dada la diversidad existente y las particularidades de cada infante es necesario atenderlos de manera individual, ajustando cada acción e intervención de forma personalizada.

Numerosos estudios han demostrado que con una atención educativa oportuna los niños y niñas con dislalia funcional, mejoran sus resultados en el proceso de aprendizaje de la lectoescritura. (Figueredo, 1984; Oriol, 2000; Cobas, 2006; Pascual, 2007; Céspedes, 2011)

Los problemas de aprendizaje de la lectoescritura pueden presentarse porque no siempre reconocemos la importancia que tiene el desarrollo del lenguaje desde edades tempranas. Santoyo, (2014)

\section{La guía metodológica como alternativa se solución.}

Se define como guía metodológica al instrumento que orienta al docente sobre los métodos y recursos que puede utilizar para cumplir sus funciones y brindar una atención educativa oportuna a los escolares. Esta guía deviene un recurso para el aprendizaje y la formación de los niños y niñas de forma progresiva, planificada, consciente y organizada. Además, proporciona datos técnicos y se enfoca en la educación a través de la participación activa de los escolares. Ron Vargas, (2019)

Las guías metodológicas, en el proceso educativo, cumplen con diversas funciones:

- Favorecen el trabajo y organización de los docentes, así como la intervención educativa.

- Estimulan el trabajo en forma grupal o individual.

- Contribuyen a desplegar la creatividad y búsqueda de información del docente, en función de las necesidades de sus estudiantes.

- Constituyen para estudiantes y docentes una base orientadora para la acción ya que especifica las actividades a desarrollar, así como la forma de hacerlo.

- Es una vía de retroalimentación para el reajuste de recursos y formas de enseñar.

- Específica, a partir de un diagnóstico de necesidades, las principales áreas de incidencia y las problemáticas que se deben solucionar.

Las funciones de la guía metodológica son: motivadora, de orientación y diálogo, facilitadora y evaluadora. (García Hernández \& De la Cruz Blanco, 2014) 
La concepción de la guía metodológica está sustentada en modelos constructivistas, y asentada en las estrategias de "aprender a aprender" y "aprender construyendo". Bengoechea, P. (2006)

Partiendo de lo señalado se plantea como Problema Científico ¿Cómo contribuir al aprendizaje de la lectoescritura en niños y niñas con dislalia funcional?

El cuestionamiento anterior conduce a asumir como objetivo del presente trabajo: Diseñar una guía metodológica que favorezca el aprendizaje de la lectoescritura en los niños y niñas con dislalia funcional.

Es importante destacar que el desarrollo de una guía metodológica que favorezca el aprendizaje de la lectoescritura en los niños y niñas con dislalia funcional, se convierte en un valor añadido para el trabajo en el subnivel básico elemental. Orienta un conjunto de actividades para niños y niñas entre 6 y 8 años de edad, constituyendo un recurso para propiciar la atención a la diversidad en las instituciones educativas, teniendo en cuenta además el papel que desempeña la familia y la comunidad educativa.

Por otra parte teniendo en cuenta los antecedentes teóricos relacionados con la dislalia funcional y su incidencia en el proceso de aprendizaje de la lectoescritura y considerando además que tanto a nivel internacional como en Ecuador es un trastorno muy frecuente que requiere de atención e intervención desde edades temprana, la autora es del criterio que la investigación es pertinente y actual. La misma contribuye a dar solución a la problemática objeto de estudio, además de favorecer los procesos de inclusión y atención a la diversidad.

\section{Metodología.}

La investigación realizada siguió un tipo de estudio mixto, donde se articulan elementos cualitativos y cuantitativos. De carácter descriptivo no experimental, con el empleo de métodos teóricos como: el histórico .lógico, el analitico-sintetico, el inductivo deductivo y el sistémico estructural funcional, análisis documental, los cuales permitieron establecer los fundamentos teóricos y modelar la propuesta. Conjuntamente se utilizó como método empírico la observación, la cual estuvo acompañada de la encuesta como técnica fundamental. Todo ello permitió corroborar el problema objeto de estudio y plantear las posibles soluciones

La investigación se realiza considerando una población integrada por 158 personas, entre padres, docentes y estudiantes según las características de los sujetos objeto de estudio. La muestra se seleccionó a partir de un muestreo no probabilístico, con carácter intencional, dirigida a un grupo 79 personas.

Con el objetivo de conocer la situación actual de la preparación que reciben los niños y niñas con dislalia funcional para enfrentar el proceso de aprendizaje de la lectoescritura, 
se aplicaron encuestas a padres de familia (25), docentes (4) y observación directa a estudiantes (50).

Los resultados de los instrumentos aplicados permitieron identificar:

\section{Fortalezas:}

- Disposición de los docentes y de los padres de familia para mejorar el trabajo con los escolares que presentan dislalia funcional.

- Disposición de los escolares para corregir la dislalia funcional.

\section{Debilidades:}

- Desconocimiento por los docentes y padres de familia de que es la dislalia funcional.

- Insuficiente preparación de los docentes para atender a escolares con dislalia funcional.

- Insuficiente orientación a la familia para contribuir a la corrección de la dislalia funcional.

\section{Discusión de Resultados.}

Las guías metodológicas son una herramienta que orientan o dirigen hacia un objetivo, ofrece una metodología a seguir en el proceso de aprendizaje.

Teniendo en cuenta lo que se establece en el currículo para el subnivel básica elemental, Ministerio de Educación (2016), se definieron los contenidos de la guía metodológica, con actividades para corregir la dislalia funcional de los escolares en este subnivel y favorecer el aprendizaje de la lectoescritura.

\section{Objetivo General:}

Proporcionar alternativas a los docentes del subnivel básica elemental para la atención educativa a los escolares que presentan dislalia funcional, que favorezcan el aprendizaje de la lectoescritura.

\section{Objetivos Específicos}

- Establecer las actividades idóneas que favorezcan la corrección de la dislalia funcional.

- Identificar estrategias que orienten el manejo de la guía con la finalidad de preparar a los escolares para el aprendizaje de la lectoescritura.

- Trabajar por medio de ejercicios prácticos y propiciar la interacción de los escolares. 
La guía metodológica es un instrumento que propone algunas actividades generales dirigidas al docente para que las utilice con sus estudiantes y así poderle dar una atención educativa oportuna y eficaz a la dislalia funcional que manifiestan estos. Pretende organizar y orientar la labor educativa del docente.

También propone diversas vías y procedimientos que el docente puede realizar. Además, orienta el tipo de actividades que se pueden efectuar con los escolares. Asimismo, presenta una variedad de ejercicios que sirven de patrón para el tratamiento.

\section{Orientaciones generales a los docentes:}

La guía metodológica propuesta consta de diferentes momentos articulados mediante diversas actividades que se pueden desarrollar no solo en la clase, sino que pueden constituir actividades a realizar por los escolares incluso fuera del horario docente. Hay diversas formas de presentación de los ejercicios, a continuación de exponen gráficamente los momentos:

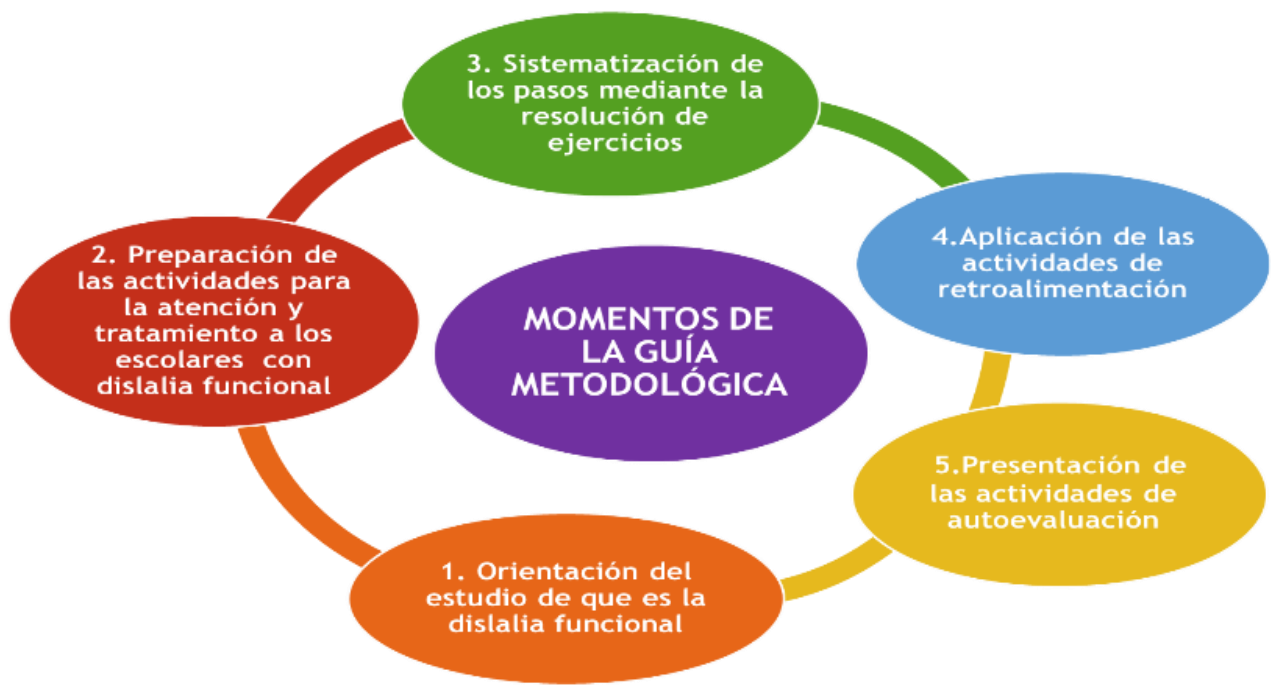

Figura 1: Momentos de la Guía Metodológica a emplear por el docente Elaborado por Mindiola, (2020)

El profesor debe seguir una metodología para trabajar con estos momentos mencionados anteriormente, le ofrecen los pasos a cumplir para garantizar el aprendizaje y su aplicación:

1. Explicar el orden para desarrollar las actividades.

2. Antes de proceder a realizar la actividad se explica y ejemplifican las acciones a desarrollar por los escolares.

3. El docente elegirá cuántos ejercicios debe hacer cada escolar.

4. Las actividades deben realizarse en contextos comunicativos, individuales y colectivos de forma oral.

Los resultados obtenidos con la propuesta, se enfocan hacia la preparación de los docentes que atienden escolares con dislalia funcional en el subnivel básica elemental, lo que favorece el proceso de aprendizaje de la lectoescritura. 
La concepción y puesta en práctica de la propuesta conduce al enriquecimiento de un marco teórico y práctico en torno a una cultura que promueve la inclusión educativa.

Los docentes se apropian de herramientas conceptuales, metodológicas y prácticas que les permiten utilizar los recursos y apoyos que requiere cada escolar con necesidades educativas especiales asociadas o no a discapacidad en las aulas. Ainscow, M. (1995)

\section{Conclusiones.}

- El estudio teórico permitió fundamentar la problemática objeto de estudio y los beneficios que trae el diseño de una guía metodológica para alcanzar mayor preparación en los docentes participantes

- El análisis de las indagaciones en la etapa de inicial corroboran las principales problemáticas en la preparación de los docentes y familias para la atención a los escolares con dislalia funcional, confirmándose la necesidad de buscar alternativas de solución al problema objeto de estudio

- El diseño e implementación de la guía metodológica como alternativa en la preparación de los docentes y sus familias, favorece la preparación de los escolares con dislalia funcional para el aprendizaje de la lectoescritura, lo que evidencia la viabilidad de la propuesta.

- La implementación de la guía metodológica contribuye a la atención oportuna a la diversidad de casos con presencia de dislalia funcional favoreciendo sus procesos de inclusión, respetando las individualidades y potencialidades presentes en cada niño independientemente a su condición.

\section{Referencias Bibliográficas}

Ainscow, M. (1995). Necesidades especiales en el aula. Ed. Narcea. Madrid.

Bengoechea, P. (2006). Aprendizajes constructivistas y no constructivistas una diferencia obligada para nuestras aulas. Aula Abierta, 23(2) 27-54.

Cabanas Comas, R. (1975). Relaciones específicas entre las dificultades en el aprendizaje de la lectura y escritura y los trastornos del lenguaje oral. Revista Cubana de Pediatría, vol. 47, No. 4-5, julio-octubre.

Céspedes, C. (2011). Trastornos del lenguaje oral. Revista Innovación y Experiencias Educativas. $8(1)$ 57-63 Recuperado de http://www.csicsif.es/andalucia/modules/mod_ense/revista/pdf/Numero_38/CRISTINA_CESP EDES_2.pdf

Cobas, C. L. (2006). La Preparación logopédica para el maestro primario. La Habana: Pueblo y Educación.

Figueredo, E. (1984). Logopedia I (Vol. I). La Habana: Pueblo y Educación.

García, I. y De la Cruz, G. D. (2014). Las guías didácticas: recursos necesarios para el aprendizaje autónomo. EDUMECENTRO, 8(15), 162-175. 
Loza, J. (2018). Adaptaciones curriculares y el aprendizaje de lectoescritura en estudiantes de educación general básica elemental del Colegio Gutenberg Schule (Tesis de maestría). Quito: Universidad Andina Simón Bolívar.

Ministerio de Educación (2016). Currículo de EGB y BGU de Lengua y Literatura. Recuperado de https://educacion.gob.ec/wpcontent/uploads/downloads/2016/03/LENGUA.pdf

Muse, C. y Delicia D. (2015). Lectura y Escritura: continuidades, rupturas y reconstrucciones. https://rdu.unc.edu.ar/bitstream/handle/11086/2308/serie\%20Unesco_vol umen\%2002_AAVV.pdf?sequence=1\&isAllowed=y

Oriol, L. (2000). Prevalencia de dislalias en los alumnus de ler año EGBde las escuela de Melincue. (Doctoral dissertación, Universidad Nacional de Rosario. Facultad de Ciencias Médicas Escuela de Fonoaudiología, Rosario. Disponible en: https://w.w.wresearchgate .net/publication/33739967

Pascual, G. (2007). La dislalia: Naturaleza, diagnóstico y rehabilitación. Madrid: Ed. Narcea.

Pérez, E. E. (2016). Un estudio de revisión sobre la dislalia y la intervención educativa. España: Ed. La Laguna.

Rodríguez, E. C. (2010). Alumnos/as con dislalia: evaluación e intervención. Revista Reflexiones y Experiencias Innovadoras en el Aula. Recuperado de http://www.didacta21.com/documentos/revista/Octubre10_Rodriguez_Cuello_El isabeth

Ron, M.E. (2019). Guía metodológica para la atención educativa a estudiantes con Disortografía en el noveno nivel de Educación General Básica (Nivel Superior) (Tesis de maestría). Milagro: San Francisco de Milagro.

Santoyo, S. (2014). La lectoescritura en la escuela primaria y sus trasformaciones ante la incorporación de las TIC. PAPELES, 6(11), 36-47. Recuperado de: http://revistas.uan.edu.co/index.php/papeles/article/viewFile/395/274

Sailema A., Maqueira, Sailema, M., \& Sailema, AD., "Estrategia metodológica, esquema corporal y actividad física adaptada: Una trilogía para la inclusión social. Revista Ciencia Digital. ISSN: 2602-8085 Vol. 3, №2.5, p. 186-196, abril - junio, 2019.DOI https://doi.org/10.33262/cienciadigital.v3i2.5.53Alonso, G. (2020), Competencias del docente en la atención a estudiantes con conductas disruptivas de 8vo año de Educación General Básica. Tesis en Opción al grado de Magister en Inclusión Educativa y Atención a la Diversidad Universidad Universidad Laica Vicente Rocafuerte. Guayaquil. ( próxima a sustentación)

Campoverde, M. (2014). Desempeño docente en el proceso de enseñanza aprendizaje en el nivel de Educación Básica de la Unidad Educativa "Belisario Quedo" en la ciudad de 
Pujilí, provincia de Cotopaxi, Año lectivo 2013-2014. Cotopaxi-Ecuador: Universidad Técnico Particular de Loja.

Cépedes, A. (2012). Niños en Pataletas.adolescentes desafiantes, Como manejar los trastornos de conductas en los hijos.Santiago de Chile: Ediciones Básicas Chile SA

Cuberes, R (2013) Causas de las conductas desafiantes en alumnos de primaria.Trabajo de fin de Grado Universidad Internacional de la Rioja.Facultad de Educación.

Duarte, J. (2017). Formación permanente de docentes en servicio, alternativa para la enseñanza y el aprendizaje de la lengua escrita en la educación básica integral. México: Universidad Rovira.

Freire, C. (2012). Factores que inciden en la presencia de conductas disruptivas de los estudiantes de $\operatorname{los} 8^{\circ}, 9^{\circ} 10^{\circ}$ años en educación básica de la Unidad Educativa experimental FAE N`3 Taura del Cantón Yaguachi durante el período lectivo 2012. Milagro: Universidad Estatal de Milagro.

Flores, G. (2018). Programa de capacitación psicológica a padres de familia para la atención de niños con conductas disruptivas de 6to EGB. Tesis en opción al grado de Magister en Inclusión Educativa y Atención a la Diversidad. Universidad Laica Vicente Rocafuerte. Guayaquil.

Espinoza, G. (2014). Desempeño docente en el proceso de enseñanza aprendzaje en el nivel de Educación Básica Superior del Centro Educativo Colegio de Bachillerato Ciudad de Portovelo. Loja: Universidad Técnica Particular de Loja.

Equipo Dide, 2017, Conductas disruptivas. La asertividad, una competencia docente primordial. http://educaryaprender.es/conductas-disruptivas-comportamiento/

Expasa. (2018). Diccionario Enciclopédico. En T. I.-t. Edición-. Barcelona: Expasa Calpe.

Jiménez, J. (2017). Conductas disruptivas en el Aula y desempeño docentes en la Institución Educativa Técnica General Roberta Leyva del Municipio de Saldaña Tolima. Ibague: Universidad del Tolima.

Jurado, P. (Coord.)(2015), Informe "Influencia de los comportamientos disruptivos en el fracaso escolar de los alumnos de ESO Hacia un modelo de intervención centrado en la institución educativa" Barcelona: UAB (Proyecto I+D-2010. EDU201020105 (subprograma EDUC)

Jurado, P \& Justiano MD(2015), "Las conductas disruptivas y los procesos de intervención en la educación secundaria” Universidad Autónoma de Barcelona (España) Cooperación Técnica Alemana (Bolivia) 
Morazán, S. (2013). Competencias docentes y su relación con el rendimiento académico en la asignatura de matemáticas en las instituciones de educación media del municipio de Danlí. Tegucigalpa: Universidad Pedagógica Nacional "Francisco Morazán".

\section{Ciencia \\ Digital \\ Edtorial}




\section{PARA CITAR EL ARTÍCULO INDEXADO.}

Mindiola Vera, C. (2020). Guía metodológica para la atención a escolares con dislalia funcional en el subnivel básica elemental. Ciencia Digital, 4(4.1), 47-57. https://doi.org/10.33262/cienciadigital.v4i4.1.1452

\section{Ciencia}

El artículo que se publica es de exclusiva responsabilidad de los autores y no necesariamente reflejan el pensamiento de la Revista Ciencia Digital.

El artículo queda en propiedad de la revista y, por tanto, su publicación parcial y/o total en otro medio tiene que ser autorizado por el director de la Revista Ciencia Digital.
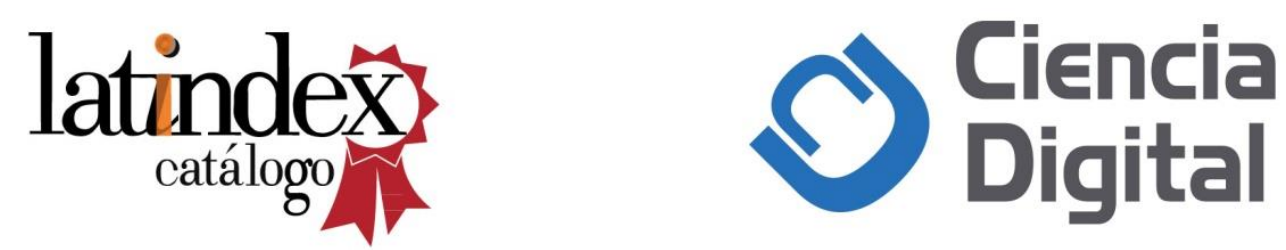